\title{
Les raisons systématiques de l'inachèvement du système fichtéen
}

The systematic reasons of the incompleteness of Fichte's system

Ives Radrizzani

\section{(2) OpenEdition}

\section{Journals}

Édition électronique

URL : http://journals.openedition.org/ref/681

ISSN : 2258-014X

\section{Éditeur}

EuroPhilosophie Editions

\section{Référence électronique}

Ives Radrizzani, «Les raisons systématiques de l'inachèvement du système fichtéen », Revista de Estud(i)os sobre Fichte [En línea], 12 | 2016, Publicado el 01 diciembre 2016, consultado el 08 septiembre 2020. URL : http://journals.openedition.org/ref/681

Ce document a été généré automatiquement le 8 septembre 2020

(c) EuroPhilosophie 


\section{Les raisons systématiques de l'inachèvement du système fichtéen}

The systematic reasons of the incompleteness of Fichte's system

Ives Radrizzani

\section{Introduction}

1 En choisissant d'utiliser pour désigner sa philosophie le terme de „Doctrine de la Science" (Wissenschaftslehre), Fichte inscrit délibérément son projet philosophique sous l'idée de science. La première occurrence connue à ce jour du terme de „Doctrine de la Science“ dans le corpus fichtéen figure dans un texte datant de février 1794 et publié il $\mathrm{y}$ a tout juste 20 ans sous le titre de Züricher Vorlesungen über den Begriff der Wissenschaftslehre, ${ }^{1}$ offrant une première version de ce que Fichte fera paraître quelques mois plus tard dans l'opuscule programmatique: Sur le concept de la Doctrine de la Science. ${ }^{2}$ Fichte écrit dans cette première version zurichoise de la Begriffsschrift que „la philosophie serait la science en soi, la science de la science en général, ou la Doctrine de la Science“ et ajoute: „Le terme de philosophie ne peut guère être conservé. Il deviendra inutilisable“. ${ }^{3}$ La version imprimée du texte précise les raisons de la caducité escomptée du terme de "philosophie“: „Cette science, pour peu qu'elle devînt jamais science, abandonnerait non sans raison un nom qu'elle a porté jusqu'ici par une modestie qui n'était pas excessive - le nom d'une prédilection, d'une marotte, d'un dilettantisme (den Namen einer Kennerei, einer Liebhaberei, eines Dilettantism ${ }^{\text {“ }} .{ }^{4}$ L'utilisation du terme de Doctrine de la Science est donc soumise à une condition: ce n'est qu'une fois que la philosophie aura véritablement été élevée au rang de science qu'elle méritera pleinement de renoncer à l'appellation sous laquelle elle est traditionnellement connue et pourra légitimement être qualifiée de „Doctrine de la Science“. Surenchérissant sur le fameux passage de la Préface de la seconde édition de la Critique de la raison pure, dans lequel Kant déplorait que la métaphysique n'ait „pas encore été jusqu'ici assez favorisée du destin pour pouvoir prendre le chemin sûr d'une science “, ${ }^{5}$ Fichte se propose de remédier au dilettantisme qu'il impute à ses prédécesseurs et de mener à bien la tâche 
identifiée mais laissée ouverte par Kant: élever la philosophie au rang d'une science rigoureuse. Fichte en est en effet convaincu: ni Kant, ni son successeur Reinhold, ne seraient parvenus à s'acquitter de cette tâche, et à l'instar de Kant qui avait eu besoin d'un Hume pour être tiré de son sommeil dogmatique, ${ }^{6}$ il aurait eu lui-même besoin d'une médiation sceptique, comme il le confesse dans la Préface à la Begriffsschrift, pour prendre la mesure de l'insuffisance de la solution proposée par ses prédécesseurs. ${ }^{7} \mathrm{Au}$ reste, écrit-il dans l'Essai d'une nouvelle présentation de la Doctrine de la Science, si Kant avait été en mesure d'apporter à la philosophie critique cet achèvement systématique, nécessaire selon lui pour élever la philosophie au rang d'une science rigoureuse, „'auteur de la Doctrine de la Science se serait épargné le travail et aurait choisi de s'attacher à une autre branche du savoir humain". ${ }^{8}$

2 En retenant la désignation de „Doctrine de la Science“ pour désigner les diverses présentations de son système, Fichte veut donner à penser qu'il aurait conféré à la philosophie cette systématicité jusqu'alors vainement recherchée et qu'il peut désormais se passer du terme désormais caduc de philosophie. Mais la multiplicité des enveloppes dans lesquelles il a présenté sa doctrine n'est-elle pas en contradiction avec le caractère de scientificité qui, selon la conception ordinaire, exige l'unité? Et chaque nouvelle présentation de la Doctrine de la Science n'est-elle pas en quelque sorte un aveu de défaite: l'indice d'une incapacité à arrêter sous une forme fixe le système un et éternel prétendument découvert? Le but de cette contribution est d'engager une réflexion sur la conception fichtéenne de système. Nous nous arrêterons en particulier au rapport entre philosophie et langage, ce qui fera l'objet de notre permière partie, et au rapport entre philosophie et vie, à quoi nous consacrerons la seconde partie.

\subsection{L'unicité de l'objet de la Doctrine de la Science}

3 En guise d'introduction à son système, Fichte s'est servi à plusieurs reprises du Bourgeois gentilhomme de Molière..$^{9}$ De même que Maître Jourdain prend soudain conscience qu'il a toujours parlé en prose, de même celui qui s'élève au point de vue philosophique, ou transcendantal, prend conscience des opérations nécessaires de son esprit et de son corps dont il s'acquitte au point de vue de la conscience naturelle sans en être conscient. L'ensemble des opérations requises pour que l'homme puisse prendre concience de lui-même constituent un système.

4 Le philosophe transcendantal n'a pas à ,inventer“ ce système, il a seulement à le "découvrir", et Fichte n'hésite pas pour illustrer son propos à établir un parallèle avec les lois de la physique: de même que le physicien n'a pas à inventer les lois de la physique, lesquelles agissent indépendamment de la connaissance que nous en avons Fichte mentionne en particulier les lois de l'électricité ${ }^{10}-$, de même le philosophe n'aurait pas à inventer les lois de la conscience, qui opèrent indépendamment de la conscience que nous en avons.

5 La Doctrine de la Science comme système vise la présentation du système de l'esprit qui est son objet. Plus précisément, la nature systématique de la Doctrine de la Science tient à la nature systématique de son objet, l'esprit humain, dont elle prétend uniquement être l'«historiographe», selon la formule bien connue de la Begriffsschrift. ${ }^{11}$

6 Le système transcendantal peut être achevé, car le nombre des conditions qui ont dû être remplies pour permettre la conscience est fini. «Achever» la philosophie revient dès lors à établir le répertoire exhaustif des actions originaires qui doivent être opérées 
pour permettre la conscience, autrement dit à énumérer l'ensemble des conditions transcendantales de la conscience.

\subsection{Multiplicité des présentations de la Doctrine de la Science}

7 Fichte n'a pas présenté sous une forme unique cet objet un qui fait l'objet de la Doctrine de la Science. Une série impressionnante de présentations s'égrènent au fil de son parcours. Il est difficile d'en fixer le nombre exact. Dans un article récent, Günter Zöller a pensé pouvoir en dénombrer dix-sept, cherchant à établir de façon un peu forcée un parallélisme avec les dix-sept quatuors à cordes de Beethoven. ${ }^{12}$ Peu importe au fond le nombre précis. Il est suffisamment important, pour que l'on puisse écarter l'hypothèse d'un tâtonnement fortuit. Fichte a délibérément choisi de multiplier les entrées dans son système, s'évertuant à modifier autant que faire se peut terminologie, structure, méthode. Selon une formule heureuse de Reinhard Lauth souvent utilisée dans ses cours, Fichte a fait œuvre de „polyglotte“. Il s'est appliqué sa vie durant à mettre en pratique le programme esquissé dans une lettre à Reinhold: „Ma théorie doit être présentée de façon infiniment variée, chacun la pensera - et devra la penser autrement, pour la penser par lui-même “ ${ }^{13}$ Hostile à toute pétrification de la lettre de son système, qui permettrait aux aveugles de l'esprit d'avoir trop facilement l'impression de pouvoir s'en rendre maitre en se dispensant de l'effort de penser par eux-mêmes, Fichte s'est plu à mettre en œuvre une technique de fluidification de la pensée qui contraigne chacun à se livrer à une véritable expérimentation mentale, sans repères trop commodes auxquels se raccrocher: „J'ai cherché autant que possible, écritil dans l'Avertissement à la première édition des Principes de la Doctrine de la Science, à éviter une terminologie fixe, qui permettrait aisément aux ,myopes de l'esprit' (Buchstäbler) de vider tout système de sa substance et de le transformer en squelette desséché“ “ ${ }^{14}$ Son œuvre, telle qu'elle se présente à nous, s'offre comme un véritable laboratoire vivant de pensée. Fichte, comme il le déclare expressément, s'est fait une "maxime“ (Maxime) de ce programme de fluidification, et indique qu'il y restera fidèle dans de futures présentations de son système..$^{15} \mathrm{Il}$ réaffirme par exemple dans la Doctrine de la Science nova methodo son intention d'éviter toute terminologie fixe, ajoutant en lançant une pointe contre Kant que c'est en adoptant le parti contraire que celui-ci se serait fait „tant d'adeptes“. ${ }^{16}$ Avant de s'indigner contre la naïveté d'un tel procédé, si contraire à notre conception de la scientificité, tout entière inscrite sous la bannière de la philologie, force est d'admirer l'étonnante vitalité d'esprit et le puissant génie dialectique à l'œuvre, qui conduit Fichte à remettre quelque dix-sept fois le travail sur le métier pour éviter de se laisser emprisonner dans quelque lettre que ce soit.

8 Moyen didactique, cette „variation à l'infini“ est-elle une fin en soi ou doit-elle être considérée comme une simple propédeutique à une présentation définitive qui fixerait pour toujours la lettre de cette philosophie „tout esprit"? Certaines déclarations de Fichte donnent à penser qu'il avait bel et bien en vue une telle présentation „finale“, suggérant que les versions de la Doctrine de la Science ne seraient que des approximations imparfaites et provisoires destinées à être supplantées par un exposé définitif, une fois les conditions réunies. C'est ainsi qu'il écrit dans l'Avertissement à la première édition des Principes de la Doctrine de la Science qu'il restera „fidèle à cette maxime [consistant à éviter autant que possible une terminologie fixe] dans de futures 
élaborations du système jusqu'à la présentation finale achevée (endliche vollendete Darstellung) de celui-ci“. ${ }^{17}$

On est en droit de s'interroger sur le statut d'une telle déclaration. Fichte n'avait-il pas quelques mois plus tôt écrit dans les Conférences sur la destination du savant que la "perfection“ (Vollkommenheit) serait le but suprême mais inaccessible de l'homme, lui assignant uniquement comme sa destination propre de s'approcher indéfiniment de ce but dans un processus de "perfectionnement à l'infini“ (Vervollkommnung ins Unendliche). ${ }^{18}$ Or on ne voit pas comment Fichte, sauf à s'affranchir de sa condition humaine, aurait jamais pu se trouver en position de présenter la version „finale achevée“ de son système, et ce qui aurait pu le motiver à tuer l'esprit de sa philosophie, auquel il tient tant, en l'enfermant dans une lettre, toute parfaite fut-elle.

Quoi qu'il en soit, le fait est que Fichte n'a jamais écrit une telle „présentation finale achevée“.

\section{I. Philosophie et langage}

\subsection{La Doctrine de la Science comme „actologie“ et l'inadéquation radicale du langage}

11 Allons plus loin et demandons-nous s'il y a des raisons systématiques qui rendent la Doctrine de la Science particulièrement perméable au danger de pétrification. Un premier élément de réponse consiste dans la nature du langage, à l'égard duquel Fichte nourrit une méfiance radicale et qui s'avère particulièrement peu approprié au caractère spécifique de la Doctrine de la Science.

Il n'est bien sûr pas possible de faire complètement l'économie du langage, véhicule par excellence de la sollicitation nécessaire à l'éveil de la conscience, instrument privilégié de communication, mais le philosophe ne doit avoir de cesse de se méfier de son pouvoir mortifère et d'en dénoncer la portée dogmatisante. Sans langage, il n'y aurait, cela va de soi, pas de philosophie, mais le langage trahit perpétuellement la philosophie, fossilise le fluide, transforme en quasi chose l'acte, et fait constamment resurgir le spectre du dogmatisme. Le langage est à la fois un outil incontournable et foncièrement inadéquat.

Sans langage, il n'y aurait pas de philosophie. La pensée s'articule en concepts, et le langage se prête en particulier à assurer une fonction logique, que Fichte s'est toujours appliqué à respecter. C'est ainsi que dans les Principes, Fichte met le plus grand soin à établir la structure formelle: même celui qui ne serait pas en mesure de procéder sur lui-même à l'expérimentation demandée, ce dont Fichte prend la peine de spécifier que ce ne saurait être le cas d'aucun être raisonnable fini, „devrait cependant en concéder la justesse formelle“. ${ }^{19}$

14 La fonction logique ne constitue toutefois que l'un des aspects du langage. La spécificité de la Doctrine de la Science est de ne pas avoir primairement affaire à des faits (Tatsachen), à des concepts, à des choses quelles qu'elles soient. En tant qu'analyse immanente des conditions de la conscience, elle ne s'occupe que d'actes (Tathandlungen). Pour utiliser une expression chère à Augustin Dumont, elle est en son

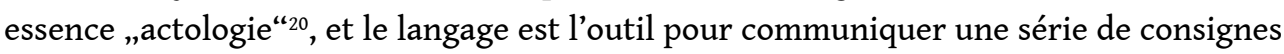
devant permettre à l'interlocuteur ou au lecteur de produire librement l'acte sollicité. 
Concernant cette dimension actologique, Fichte exprime de très fortes réserves à l'encontre du langage, qui, par une pente naturelle, aurait tendance à transformer l'acte en chose, à faire prévaloir l'être sur la genèse, à briser la dynamique de la pensée. La construction rigoureusement géométrique adoptée pour la présentation des principes dans la Grundlage der gesamten Wissenschaftslehre paraît répondre à certains égards au critère de scientificité revendiqué par Fichte pour son système. En réalité, ce n'est pas fausse modestie si Fichte a très rapidement marqué son insatisfaction à l'égard de la forme adoptée dans cette première version de son système. Et s'il se fixe pour but de produire une philosophie scientifique qui puisse se mesurer aux mathématiques, ${ }^{21}$ cela n'implique nullement que la scientificité recherchée doive se calquer sur le modèle des mathématiques. Sans doute, la preuve en géométrie, fondée sur une construction supportée par l'intuition, fera toujours, pour Fichte, y compris dans sa période dite tardive, figure de modèle. Mais si les mathématiques, et plus particulièrement la géométrie, offrent un exemple réussi de formalisation appuyée sur une intuition, qui leur procure la rigueur et la certitude et dont la philosophie, à l'instar des autres sciences, a intérêt à s'inspirer si tant est qu'elle veuille s'engager sur la «sûre route de la science», elles ne détiennent nullement le monopole du modèle de formalisation correspondant au critère formel de la science, pas plus qu'elles ne détiennent le monopole de la certitude correspondant à son critère matériel et, en tant que science dérivée, doivent être strictement subordonnées à la Doctrine de la Science qui, comme science première, doit fonder aussi bien leur forme que la certitude de leur principe premier. Fichte reproche en particulier aux Principes le caractère artificiel de la démarche adoptée, où structure formelle et mise en œuvre du pouvoir de vision se trouvent dissociés, et il s'emploiera dans les exposés ultérieurs à remédier à ce défaut, dû selon lui à une trop grande complaisance à l'égard de la tradition. C'est ainsi qu'il renonce dans la Doctrine de la Science nova methodo à la divison entre théorie et pratique et présente, „selon une démarche bien plus naturelle“ la „philosophie même“, théorie et pratique liées. ${ }^{22}$

16 L'important remaniement méthodique opéré dans la Doctrine de la Science nova methodo semble bien aller dans la direction de cette „actologie“ qui constitue l'essence de la Doctrine de la Science, et c'est en ce sens que Fichte peut parler d'une démarche plus naturelle. Toutefois, l'amélioration apportée ne permet pat d'éliminer le danger de réification inhérent selon lui au langage, et c'est pourquoi il persévère dans son refus à mettre en place une terminologie fixe.

Pour écrire la „présentation finale achevée“ dont il a été question, il faudrait trouver le moyen d'inventer un nouveau langage qui ne donne aucune prise à la tendance réifiante du langage ordinaire, un langage purement performatif où l'esprit ne vienne pas butter contre la lettre.

\subsection{La ruse du langage et la dimension ironique}

L'une des techniques développées par Fichte pour pallier au défaut intrinsèque du langage consiste en une stratégie de communication qu'il m'est arrivé à plusieurs reprises de qualifier de „ruse du langage“, par analogie avec la ruse de la raison. Cette technique, présente à travers tout le corpus fichtéen et déjà clairement identifiée par Xavier Léon en son temps, ${ }^{23}$ mérite bien ce qualificatif de „ruse du langage“ car elle vise à miner en quelque sorte de l'intérieur le discours de celui à qui il s'adresse. Par le 
truchement de cet artifice langagier, Fichte entre en quelque sorte dans le jeu de ses adversaires ou de ses interlocuteurs, emprunte par exemple leur terminologie, non pas qu'il veuille signifier par là une quelconque concession ou un infléchissement de sa pensée, mais parce qu'il estime qu'il s'agit du moyen privilégié pour se faire entendre d'eux et pour les amener dialectiquement à la Doctrine de la Science. Face aux Romantiques il parlera en romantique; dans le contexte de l'occupation napoléonienne il mimera un langage nationaliste; en Loge il adoptera la phraséologie maçonnique; face à Jacobi il deviendra un philosophe de la vie; face à Schelling et à Hegel il singera un discours ontologique. ${ }^{24}$ Cette utilisation curieuse du langage avait déjà frappé les contemporains; c'est ainsi que Jean Paul disait de la Destination de l'homme qu'elle était écrite dans un "chiffre destructeur" (vernichtende Chiffre), parce que, „pour les lecteurs exotériques“, elle „signifie toujours le contraire“ ${ }^{25}$ Le langage fichtéen est creusé d'une dimension ironique ${ }^{26}$ invitant à briser l'univocité de la lettre et nécessitant une lecture dynamique. La lettre ne se donne pas simplement. Elle convie à un acte interprétatif censé ouvrir l'œil de l'esprit. Mais ce procédé langagier qui exige de la part de l'interprète un sens aiguisé de la dialectique, a été la source d'innombrables malentendus. Doit-on s'imaginer que dans la „présentation finale achevée“, Fichte aurait supprimé cette dimension ironique qui fait l'originalité de sa contribution, du point de vue de la stratégie de communication, et dont le but est notamment de contribuer à remédier à la tendance réifiante du langage ordinaire, mais toujours liée à une situation ad hoc et donc apparamment peu compatible avec la prétention éternitaire d'une présentation „finale“?

\section{II. Philosophie et vie}

\subsection{L'ouverture sur la vie}

Il y aurait sans doute beaucoup à dire sur la conception fichtéenne du langage, mais la difficulté à trouver un langage adapté au caractère de la Doctrine de la Science comme actologie n'est pas l'unique raison de l'inachèvement du système fichtéen. Une raison plus profonde tient à la forme même de la Doctrine de la Science. Système clos en tant que description de la structure de la conscience, elle est en même temps un système ouvert. Reinhard Lauth a très bien montré comment la philosophie transcendantale, faisant fond sur la liberté qui est, par essence, non déductible, reconnaît a priori et par principe que la réalité dans sa totalité comprend une partie non déductible qui n'est saisissable qu'a posteriori. Si la philosophie en tant que Doctrine de la Science peut être achevée, c'est que, par principe, elle ne prétend pas épuiser le concret dans sa concrétude et se meut uniquement sur le plan des principes. Autrement dit, elle procède à une déduction strictement a priori. Se mouvant sur le plan de l'a priori, elle ne peut pas déduire l'a posteriori. En revanche, ce qu'elle peut déduire, c'est qu'il doive y avoir de l'aposteriori. Pour l'exprimer dans les termes de Lauth, la déduction transcendantale des conditions de la conscience met en lumière la nécessité qu'il y ait du „concret“", mais ne peut pas déduire le „concrètement concret“", le concret dans sa concrétude. ${ }^{27}$

Or, avant d'être philosophe, le philosophe est d'abord un être immergé dans la vie, historiquement engagé dans une situation unique, répondant aux sollicitations spécifiques qui lui ont été adressées et qui seules lui ont permis de s'élever à la 
conscience. La vie n'est pas un appendice facultatif qui vient s'ajouter au système, c'est au contraire le système qui vient toujours se développer sur fond de vie. Le philosophe ne peut pas se trouver sans se trouver engagé dans un tissu social d'une extrême complexité, fait d'un jeu de libres entre-sollicitations non déductibles qui offrent une matière infinie à la tâche de rationalisation qu'il s'impose librement.

La Doctrine de la Science n'est pas et ne prétend pas être une philosophia perennis, contrairement à ce que certains de ses interprètes les plus distingués ont pu affirmer. Elle n'est pas une philosophia perennis et, d'une façon plus générale, une philosophie de l'absolu, et si l'absolu est appelé à jouer un rôle important en elle, c'est au titre de principe d'explication de la conscience, du point de vue de la finitude qui est le sien, parce qu'elle est philosophie transcendantale, soucieuse de ne pas transcender les limites de la conscience et de produire la genèse de l'hors-conscience admis par le point de vue de la conscience commune.

La formidable dialectique à l'oeuvre dans les Principes a précisément pour résultat le plus remarquable de montrer que le sens de l'absoluité du premier Grundsatz ne devient clair que dans la partie pratique, une fois ce dernier ramené au statut d'une „idée“ de la raison.

Mettre l'accent sur la partie principielle du système et sur sa clôture, ce qui relève à mon avis d'une fascination pour une conception pérenne de la philosophie, à laquelle Fichte, on l'a vu, n'a lui-même pas toujours été insensible, est une tendance fortement représentée dans la tradition exégétique, mais revient à oublier cet ancrage dans la vie, si emphatiquement souligné par Fichte lui-même et que le système, malgré tout l'achèvement auquel il peut par ailleurs prétendre, est à l'infini incapable de résorber entièrement et qui, par conséquent échappe à l'infini à sa volonté de contrôle et d'arraisonnement. Exprimé négativement: l'ouverture est le défaut de la cuirasse, le point faible du système. Exprimé positivement: l'incontrôlabilité est la raison pour laquelle il y aura toujours besoin de la philosophie.

\section{Conclusion}

Nous sommes partis de la constatation d'une étonnante discrépance entre l'unité de la science recherchée et la multiplicité des présentations que Fichte a livré de cette science une, ce qui nous a amené dans un premier temps à nous intéresser au problème du langage. Malgré la déclaration explicite de Fichte évoquant la possibilité d'une présentation finale définitive de la Doctrine de la Science, il ne nous a pas été possible d'identifier un langage particulier qui convienne de façon privilégiée à l'exposé de l'édifice scientifique. Au contraire, il est apparu que le langage (en tant que, par son inscription dans le monde phénoménal, il comporte nécessairement une part de lettre et cela est $a$ fortiori valable du langage écrit) se trouve radicalement inapproprié pour rendre compte de la Doctrine de la Science qui, en tant qu'actologie, est tout esprit. L'unique moyen dont dispose le philosophe pour pallier à ce défaut inhérent à l'essence même du langage est de développer des stratégies de communication aptes à en démasquer la tendance ontologisante. L'un des dispositifs mis en place par Fichte pour faire apparaître le décalage entre esprit et lettre est le procédé de distanciation ironique instauré par le recours à la technique de la ruse du langage. L'important n'étant pas la lettre mais l'esprit, peu importe au fond la lettre utilisée, elle devra toujours signifier sa défaillance, renvoyer en négatif à l'esprit qu'elle ne peut que 
trahir. Même si Fichte a pu suggérer la possibilité d'élaborer une version définitive de son système, la Doctrine de la Science nous paraît nécessairement devoir rester inachevée.

Dans un second temps, nous nous sommes intéressés au rapport entre philosophie et vie et nous sommes parvenus à la conclusion que, même si l'on admettait que la Doctrine de la Science puisse parvenir à un achèvement sur le côté des principes, elle comprend par principe une ouverture sur la vie qui brise cet achèvement. La philosophie est constamment et inéluctablement renvoyée à la vie. La clôture est nécessairement un but qu'elle se fixe en point de mire. Mais la clôture est un but que nécessairement elle n'atteindra jamais, sauf à s'affranchir des lois de la finitude. La philosophie fichtéenne est radicalement une philosophie de la finitude, de l'existence, une philosophie qui s'enracine dans la vie, une philosophie du risque et de l'engagement, devant laquelle s'ouvre une tâche infinie. Ce n'est pas un hasard si Fichte n'a jamais produit l'exposé définitif un temps envisagé. On peut se livrer à un petit jeu de devinettes : et s'il avait vécu plus longtemps, y serait-il jamais parvenu ? Ma thèse est que sa philosophie est foncièrement réfractaire à toute clôture définitive, et cela pour des raisons d'ordre systématique. Le philosophe est dans le labyrinthe de la vie, et ce labyrinthe - belle lapalissade - est une impasse sans issue dont il ne sortira jamais. Mais la tâche infinie qui s'ouvre devant lui, loin d'être une faiblesse, est tout au contraire sa force. C'est le prix à payer pour rester une philosophie de la finitude, dénonçant toutes les impostures de la raison visant un arraisonnement définitif du réel et une mise sous tutelle de la vie. C'est pour une raison systématique que la philosophie fichtéenne est et demeure un système inachevé. Cet inachèvement, impliqué par l'ouverture sur la vie, est au demeurant l'unique inachèvement possible compatible avec l'idée de Doctrine de la Science conçue comme système clos des conditions transcendantales de la conscience. Plus précisément, abstraction faite de la question du langage, l'achèvement spécifique de la Doctrine de la Science est de ne point tolérer d'autre inachèvement que l'ouverture sur la vie requise par le système.

\section{NOTES}

1. FICHTE, J. G., Züricher Vorlesungen über den Begriff der Wissenschaftslehre. Februar 1794. Nachschrift Lavater, ed. Erich Fuchs, Neuried: Ars Una, 1996.

2. FICHTE, J. G, Ueber den Begriff der Wissenschaftslehre oder der sogenannten Philosophie, als Einladungsschrift zu seinen Vorlesungen über diese Wissenschaft, Weimar 1794.

3. RADRIZZANI, I., „La ,Première' Doctrine de la Science de Fichte. Introduction et traduction“ [= Cours zurichois], in : Archives de Philosophie, 60/4 (1997), pp. 615-658, 641 (ZV, GA IV/3, 22).

4. $C f$. „Sur le concept de la Doctrine de la Science ou de ce que l'on appelle philosophie“, in: Fichte, Essais philosophiques choisis (1794-1795) [= Concept], trad. L. Ferry et A. Renaut, Paris 1984, p. 36 (trad. revue; BWL, GA I/2, 117 sq.).

5. Critique de la raison pure, trad. J.-L. Delamarre et F. Marty, in: E. Kant, CEuvres philosophiques [= OP], vol. I, Paris 1980, p. 738 (B XIV). 
6. Cf. KANT, E., Prolégomènes à toute métaphysique future qui pourra se présenter comme science, trad. J. Rivelaygue, in: OP, vol. II, Paris 1985, p. 23 (AA IV, 260).

7. Cf. Concept, p. 19: „L'auteur de cet essai fut, par la lecture de nouveaux sceptiques, particulièrement de l'Enésidème et des excellents écrits de Maimon, pleinement convaincu de ce qui pour lui avait déjà été extrêmement vraisemblable: que la philosophie, même à travers les plus récents efforts des hommes les plus pénétrants, n'a pas encore été élevée au rang d'une science évidente.“ (BWL, GA I/2, 109).

8. FICHTE, J. G, „Seconde introduction à la Doctrine de la Science“, in: J. G. Fichte, CEuvres choisies de philosophie première. Doctrine de la Science (1794-1797), (=OCPP) trad. A. Philonenko, Paris 1980, p. 283 (trad. revue; ZE, GA I/4, 230).

9. Ibid., OCPP, p. 294 (ZE, GA I/4, 247). Cf. en outre FICHTE, J. G., „Cours sur les aphorismes de Platner“, in: Lettres et témoignages sur la Révolution française, trad. I. Radrizzani, p. 242 (GA II/4, 173) et „Cours de logique et de métaphysique“, ibid., p. 243 (GA IV/1, 401).

10. Cf. Cours zurichois, p. 654 (ZV, GA IV/3, p. 38).

11. Concept, p. 66 (GA I/2, 147).

12. zöLLER, G., „Parallelleben. Fichte und Beethoven“, in: RADRIZZANI, I., (éd.), Fichte und die Kunst, Fichte-Studien, vol. 41 (2014), p. 293.

13. Lettre à Reinhold du 21 mars 1797, GA III/3, 57.

14. FICHTE, J. G., Les Principes de la Doctrine de la Science (1794-1795) [= Principes], in: OCPP, p. 14 (trad. revue; GWL, GA I/2, 252).

15. Ibid.

16. FICHTE, J. G., La Doctrine de la Science Nova Methodo. Suivi de Essai d'une nouvelle présentation de la Doctrine de la Science, trad. I. Radrizzani, Lausanne 1989, p. 60 (WLnm-K, GA IV/3, 339).

17. Principes, p. 14 (GA I, 2, 252).

18. FICHTE, J. G., Conférences sur la destination du savant (1794), trad. J.-L. Vieillard-Baron, Paris 1980, p. $42(\mathrm{BG}, \mathrm{GA} I / 3,32)$.

19. GWL, GA I/2, 390; OCPP 128.

20. DUMont, A., L'opacité du sensible chez Fichte et Novalis - Théories et pratiques de l'imagination transcendantale à l'épreuve du langage, Grenoble : Millon, 2012, par exemple p. 56 sq.

21. Cf. la lettre de Fichte à F. V. Reinhard du 15 janvier 1794, GA III/2, 39sq.

22. DSNM, p. 49 (WLnm-H, GA IV/2, 17).

23. LÉON, X., Fichte et son temps, 3 vol., Paris, Colin, 1922-1927.

24. Concernant le subvertissement du discours nationaliste dans les Discours à la nation allemande, cf. mon article: „Ist Fichtes Modell des Kosmopolitismus pluralistisch?“, in: Fichte-Studien, 2 (1990), pp. 7-19; concernant le subvertissement du discours maçonnique, $c f$. mon „Introduction“, in: J.G. FICHTE, J., G., La philosophie de la Maçonnerie et autres textes, trad. I. Radrizzani et Fawzia Tobgui, Paris, Vrin, 1995, pp. 7-54.

25. . Lettre de Jean Paul à Karl August Böttiger du 11 mars 1800, citée in : Erich Fuchs (éd.), Fichte im Gespräch - Berichte der Zeitgenossen, Stuttgart-Bad Cannstatt: Frommann-Holzboog, 8 vol., 1978-2012, vol. 2, p. 303.

26. A l'égard de l'influence de Fichte sur du concept d'ironie romantique, $c f$. mon article: „Genèse de l'esthétique romantique : De la pensée transcendantale de Fichte à la poésie transcendantale de Schlegel“, in : Revue de Métaphysique et de Morale, 101/4 (1996), pp. 471-498.

27. Cf. par exemple R. Lauth, „Le véritable enjeu des Discours à la nation allemande de Fichte“, in : I. Radrizzani (Ed.), Approches de Fichte, in: Revue de Théologie et de Philosophie, 123 (1991), p. 269. 


\section{RÉSUMÉS}

For some years Fichte seems to have envisioned the possibility of presenting a definitive and final version of his Science of Knowledge. This article develops two reasons why such a project is structurally impossible. On the one hand, Fichte, inspired by the tradition of negative theology, challenges the capacity of language of being pure performativity: it always involves an element of letter which prevents it from being all spirit. On the other hand, the opening of the system to life implies a kind of incompleteness which is the only possible and consistent with the idea of the Doctrine of Science (understood as closed system of transcendental conditions of consciousness). More precisely, the specific completion of the Doctrine of Science cannot tolerate another kind of incompletion but that resulting of the opening to the life required by the system.

INDEX

Keywords : life, system, incompleteness

\section{AUTEUR}

\section{IVES RADRIZZAN}

Académie Bavaroise des Sciences 